\title{
Patient Perceptions of Unmet Medical Need in Rheumatoid Arthritis: A Cross-Sectional Survey in the USA
}

\author{
Christine Radawski - Mark C. Genovese - Brett Hauber · \\ W. Benjamin Nowell - Kelly Hollis · Carol L. Gaich - Amy M. DeLozier · \\ Kelly Gavigan · Maria Reynolds · Anabela Cardoso · Jeffrey R. Curtis
}

Received: June 20, 2019 / Published online: August 6, 2019

(C) The Author(s) 2019

\section{ABSTRACT}

Introduction: Many rheumatoid arthritis (RA) patients do not achieve their treatment goals and experience symptoms that affect psychosocial outcomes and daily activities. This study aimed to identify and quantify the unmet needs perceived by US patients with RA

Enhanced Digital Features To view enhanced digital features for this article go to: https://doi.org/10.6084/ m9.figshare.8982461.

Electronic Supplementary Material The online version of this article (https://doi.org/10.1007/s40744019-00168-5) contains supplementary material, which is available to authorized users.

C. Radawski $(\bowtie) \cdot$ C. L. Gaich · A. M. DeLozier .

A. Cardoso

Eli Lilly and Company, Indianapolis, IN, USA

e-mail: c.radawski@lilly.com

M. C. Genovese

Stanford University, Palo Alto, CA, USA

B. Hauber · K. Hollis · M. Reynolds

RTI Health Solutions, Research Triangle Park, NC, USA

W. B. Nowell · K. Gavigan

Global Healthy Living Foundation, Upper Nyack, NY, USA

J. R. Curtis

University of Alabama at Birmingham, Birmingham, AL, USA currently taking a disease-modifying antirheumatic drug (DMARD).

Methods: A cross-sectional, web-based survey was conducted with RA patients recruited through CreakyJoints, an online patient support community, and ArthritisPower ${ }^{\circledR}$, an online patient research registry, from December 2017 to January 2018. Participant patients were aged $\geq 21$ years, failed $\geq 1$ DMARDs, and were receiving their current $\operatorname{DMARD}(\mathrm{s})$ for $\geq$ 6 months; they answered 50 questions about treatment history, RA symptoms, and flares and completed the Rheumatoid Arthritis Impact of Disease (RAID) questionnaire and the Treatment Satisfaction Questionnaire for Medication (TSQM). Treatment satisfaction was defined by a TSQM global satisfaction score $\geq 80$.

Results: Of 415 patients screened, 258 (62\%) were eligible and completed the survey; $87 \%$ were women, and $87 \%$ white, with mean (SD) age of 54.5 (11.4) years. A total of 232 patients (90\%) had current or past biologic DMARD (bDMARD) use, with $67 \%$ currently on a bDMARD, $65 \%$ on $\geq 1$ conventional synthetic DMARD, and $40 \%$ on methotrexate. Forty-three percent of patients reported daily/almost daily use of prescription pain medications, and $44 \%$ reported a current flare. Mean (SD) TSQM scores were 59 [20] for effectiveness, 59 [26] for side effects, 72 [18] for convenience, and 65 [21] for global satisfaction. The mean (SD) RAID overall score was $5.1(2.0)$ on a 0-10 scale. Only $26 \%$ (67 patients) were satisfied with their RA 
treatment. Patients not satisfied with treatment reported higher RAID scores overall and by domain, and approximately half reported a current flare.

Conclusions: Results from this real-world survey suggest that three-fourths of RA patients are not satisfied with treatments, which include bDMARDs. Patients continued to experience bothersome symptoms that impacted their daily activities and life. There remains a need for improved disease management among currently treated RA patients.

Funding: Eli Lilly and Company (Indianapolis, IN, USA).

Keywords: Patient-reported outcomes; Rheumatoid arthritis; Survey research; Satisfaction; Unmet need

\section{INTRODUCTION}

For patients with rheumatoid arthritis (RA), a number of effective treatment options are available to improve signs and symptoms and inflammation, reduce joint damage progression, and subsequently improve patients' health-related quality of life (QoL) and their ability to perform daily activities. Methotrexate (MTX) is the first-line treatment recommended for patients with RA and is often administered alone or in combination with other conventional synthetic disease-modifying antirheumatic drugs (csDMARDs) [1]. Patients who do not respond adequately to these treatment regimens may be treated subsequently with a biologic DMARD (bDMARD) or targeted synthetic DMARD (tsDMARD), with or without concomitant MTX. In current clinical practice, a tumor necrosis factor inhibitor (TNFi) is often initiated as the first advanced therapy option [1]. With the growing availability of effective targeted therapies, the management and outcomes of RA patients have improved significantly over the past decade. However, many patients still do not achieve the current recommended treatment goals of remission or low disease in clinical trials and clinical practice [2-4]. In a recent literature review by Taylor et al., it was noted in clinical studies that many patients may continue to experience RA symptoms, such as pain and disability [4]. While these symptoms may be coming from past joint structural damage (as disability) and not be related to the actual improper control of the disease, these observations suggest unmet needs remain for many patients [4].

In clinical practice, assessment of the disease and treatment adjustments based on shared decision-making between rheumatologists and their patients are core pillars of the treat-totarget strategy for RA [1]. Yet, its implementation can be difficult, and one reason is the discrepancy between rheumatologists and their patients on the assessment of what is relevant disease activity and its impact on the desired treatment and treatment goals [5]. Consequently, understanding the patient perspective is becoming increasingly important in the clinical setting. A number of studies have been conducted to elicit patient attitudes and expectations on treatment outcomes and preferences for mode of RA treatment administration [6-11]. These studies, however, did not discern the extent to which patient needs and goals are being met with current treatments.

The goal of this study was to identify, characterize, and quantify the following: satisfaction with current RA treatment, the current unmet needs perceived by patients with RA in the US, the symptoms of RA that are most bothersome to patients, and the impact of symptoms on function and QoL that may lead patients to need alternative treatments. Specifically, we developed a survey to measure patients' current experience and self-assessment in key domains of RA that Taylor et al. identified as areas of potential unmet need: pain, physical function, mental function, fatigue, social function, sexual function, the ability to work, and overall well-being [4].

\section{METHODS}

\section{Study Design}

This study was a cross-sectional, web-based survey of patients with RA who were enrolled in ArthritisPower ${ }^{\circledR}$, an online patient research registry (https://arthritispower.creakyjoints.org/). 
ArthritisPower ${ }^{\circledR}$ is one of 20 national patient registries funded by the nonprofit Patient-Centered Outcomes Research Institute (PCORI). ArthritisPower $^{\mathrm{TM}}$ is the research arm of a wider online support community for patients with RA and other types of arthritis called CreakyJoints. Patients were recruited via an e-mail invitation that contained information about the webbased survey and a unique link to it. The sampling frame was designed to achieve diversity across gender, race, and education by oversampling men, diverse race/ethnicities, and patients with a high school education or less. A sample size of 200 respondents was targeted for the study; no formal power calculations were performed.

Patients completed screening questions (described below) to determine their eligibility prior to entry into the study. Those who completed the survey were compensated $\$ 25$. The study was reviewed by the Schulman Institutional Review Board (IRB) and was determined to be exempt. The study was conducted in accordance with the ethical principles that have their origin in the Declaration of Helsinki and that are consistent with Good Pharmacoepidemiology Practices and applicable laws and regulations. Informed consent was obtained from all individual participants included in the study. Study data collection was from December 2017 to January 2018.

\section{Study Population}

Eligible patients were $\geq 21$ years of age, had the ability to read and write in English, and had a self-reported clinician diagnosis of RA. Additionally, patients responded to a series of questions about current and prior medications that indicated they had been treated with $\geq 1$ prior DMARD for treatment of RA and were on at least a second DMARD medication, alone or in combination, and stable for at least 6 months at the time of the survey.

\section{Survey Measures}

The survey consisted of 50 closed-ended questions. Topics included demographics, clinical and disease characteristics, and treatment history. The survey also contained two validated instruments. The first, Treatment Satisfaction Questionnaire for Medication (TSQM, Version II), consists of 11 questions $[12,13]$. The TSQM provides a validated score for four subscales: effectiveness, side effects, convenience, and global satisfaction. Treatment satisfaction was measured using a cutpoint global satisfaction score $\geq 80$, which has been shown in past studies to be correlated with high medication adherence $[14,15]$. The second validated instrument was the Rheumatoid Arthritis Impact of Disease (RAID) [16]. The RAID is a composite index composed of seven domains: pain, function, fatigue, physical well-being, psychological well-being, sleep disturbances, and coping. Each domain is a $0-10$ numeric rating scale (NRS), with higher scores representing greater impact of RA, and domains were weighted when calculations were performed. The total RAID score has a range from 0 to 10 , with 10 representing worst health and a score of $\leq 2$ representing an acceptable symptom state [17]. Additionally, Salaffi et al. have proposed the following cutoffs to classify disease activity using the RAID: $\leq 3$ for remission, $>3$ and $\leq 4$ for low disease activity, $>4$ and $\leq 6$ for moderate disease activity, and $>6$ for high disease activity [18]. For individual RAID domains, the following cutpoints were considered to represent symptom severity: 0 for no issues, 1-3 for mild, 4-7 for moderate, and 8-10 for severe [19].

A series of questions assessed additional symptoms and impact of RA that were not captured by the RAID; specifically, questions addressed stiffness, swelling, mental well-being, social well-being, sexual well-being, work/ school disability, and independence. Each question was an NRS, with scores ranging from 0 (no difficulty) to 10 (extreme difficulty). Scores $\geq 4$ were considered moderate to severe [19]. Patients were also presented with a set of RA symptoms (pain, fatigue, stiffness, and swelling) and asked to identify and rank the two symptoms that were most bothersome. Additionally, patients were presented with a set of impacts of RA symptoms (difficulties with physical functioning, thinking/memory, sexual 
functioning, work/school, sleeping, independence, emotions/moods, and interference with social/leisure activities) and were asked to identify and rank the two impacts that were most bothersome. Patients also were asked to report on current flares ("Are you currently having an RA flare?" Yes/No), which represents the patient's perceived RA activity and their interpretation of what flare means. Additional questions related to flares were the number of flares within the prior 30 days ("In the past 30 days, how many RA flares have you had? Please use your best estimate if you are not sure"), and the worst flare in the 30 days using a 0-10 NRS ( 0 for no flare to 10 for the worst flare). A standard definition of a flare was not provided and was left to the discretion of the patient, as has been used in some past studies $[20,21]$. Patients were also asked, "Please state how much you agree or disagree with the following statement. There are enough medication choices available for my doctor and me to manage my RA well." Patients rated their responses with a five-point Likert scale from "strongly agree" to "strongly disagree."

\section{Data Analyses}

No imputation was performed for missing data, except for the TSQM and RAID instruments. For the TSQM, scores for each domain were computed by adding all items in each domain and then transforming the score into a value ranging from 0 to 100, with 0 representing complete dissatisfaction and 100 maximum satisfaction. If responses were missing from more than one item in the effectiveness or global satisfaction domain, a domain score was not calculated $[12,13$.$] For the RAID, if one of the seven NRS$ values was missing, the mean value of the other NRS values was calculated, and this value was imputed for the missing NRS. If two or more of the NRS values were missing, the RAID was considered as a missing value [22].

Descriptive analyses were used to summarize the survey responses for the overall sample. In addition, descriptive analyses of treatment satisfaction and of the RAID were conducted for the following subgroups: patients who reported currently taking a bDMARD and reported experience with another bDMARD previously; patients who had been on RA treatment for 1 to $<5$ years or on treatment for $\geq 5$ years; and stratification of the sample by patients who reported treatment satisfaction and those who did not (global score $\geq 80$ vs. $<80$ ). All analyses were conducted with SAS software, version 9.4 (SAS Institute Inc., Cary, NC, USA).

\section{RESULTS}

\section{Patient Demographics and Characteristics}

Of 415 patients who completed the screening questions, 258 (62\%) patients were eligible and completed the survey; six patients were eligible and did not fully complete the survey. Their partial data, however, are included in the analysis. The sample selection flow chart is provided in Supplemental Fig. 1. The majority of patients were female $(87 \%)$ and white $(87 \%)$. The mean (SD) age was 54.5 (11.4), and the ages ranged from 24 to 81 years. Approximately $1 \%$ of the patients had been diagnosed with RA for $<1$ year, and among the most common comorbid conditions that patients reported were osteoarthritis (52\%), mental health (44\%), muscle or bone (39\%), and other autoimmune (34\%) conditions. The demographics of the sample were consistent with the patient populations that appear in the literature (Supplemental Table 1) [23].

Most of the patients $(90 \%, n=232)$ had past or current experience with a bDMARD for RA, and $67 \%(n=174)$ of patients reported that they were currently taking a bDMARD. Among the 58 patients who previously took a bDMARD but were not currently taking a bDMARD, 28 (48\%) reported taking a tsDMARD (tofacitinib). Additionally, 168 patients (65\%) reported currently taking at least one csDMARD, which includes MTX; and 103 patients (40\%) reported currently taking MTX (Supplemental Table 2). Furthermore, 103 patients (41\% of 252 respondents) reported daily or almost daily use of overthe-counter treatments for pain, and $43 \%$ ( $n=109$ of 252 respondents) of patients reported daily or almost daily use of nonsteroidal anti-inflammatory drug (NSAID) or opioid 


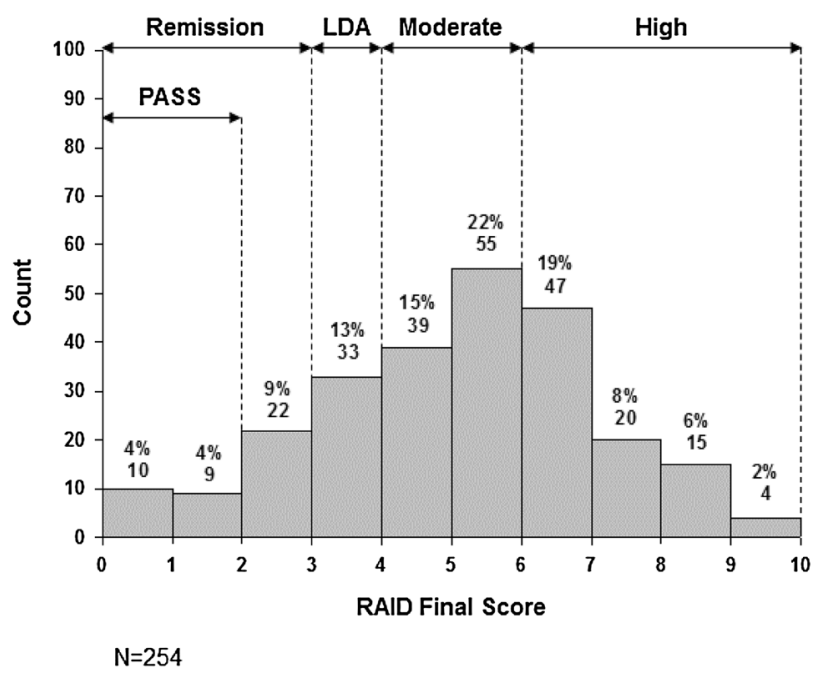

Fig. 1 Frequency of Rheumatoid Arthritis Impact of Disease (RAID) final scores. LDA low disease activity, PASS patient acceptable symptom state

prescription treatments for pain relief. Additional data on patient characteristics can be found in Supplemental Table 2.

\section{Treatment Satisfaction}

The mean (SD) TSQM scores were 59 [20] for effectiveness, 59 [26] for side effects, 72 [18] for convenience, and 65 [21] for global satisfaction.

Using the cutpoint of a global satisfaction score $\geq 80$, only $26 \%(n=67)$ were classified as being satisfied with treatment.

\section{Rheumatoid Arthritis Impact of Disease (RAID)}

The mean (SD) total RAID score was 5.1 (2.0). The distribution of the RAID total scores is presented in Fig. 1. Only 7\% $(n=19)$ met the patient acceptable symptom state with a total score of $\leq 2$, as defined by Dougados et al. [17] Using the proposed cutoffs for disease activity from Salaffi et al. [18], 16\% $(n=41)$ were in remission, $13 \%(n=33)$ in low disease activity, $37 \%(n=95)$ in moderate disease activity, and $33 \%(n=85)$ in high disease activity despite current treatment.

Fatigue, sleep disturbances, and pain were the RAID domains with the highest (worst) mean scores (Fig. 2). The symptoms of RA with the greatest percentages of patients reporting moderate-to-severe impact on their life were fatigue $(82 \%)$, pain $(76 \%)$, and physical wellbeing $(75 \%)$.

\section{Additional Symptoms and Impacts of RA}

Among the questions evaluating symptoms and impacts of RA that were not captured by the RAID, $70-80 \%$ of patients reported moderate-to-severe stiffness as a symptom and moderate-to-severe impact of RA on sexual well-being. The percentage of patients reporting moderate-to-severe symptoms or impacts are presented in Fig. 3.

Among the four symptoms patients were asked to rank as most bothersome, 51\% indicated pain, $36 \%$ indicated stiffness, $11 \%$ indicated fatigue, and $2 \%$ indicated swelling. Among the eight RA domains that patients were asked to rank as the most bothersome, the most commonly reported were physical function $(49 \%)$, sleeping $(17 \%)$, and difficulties with thinking or memory (12\%).

\section{Flares}

A current flare was reported by $44 \%$ of patients. Patients in the study reported a mean (SD) of 4.3 (7.0) flares over the past 30 days. When asked to report on the worst flare in the 30 days 


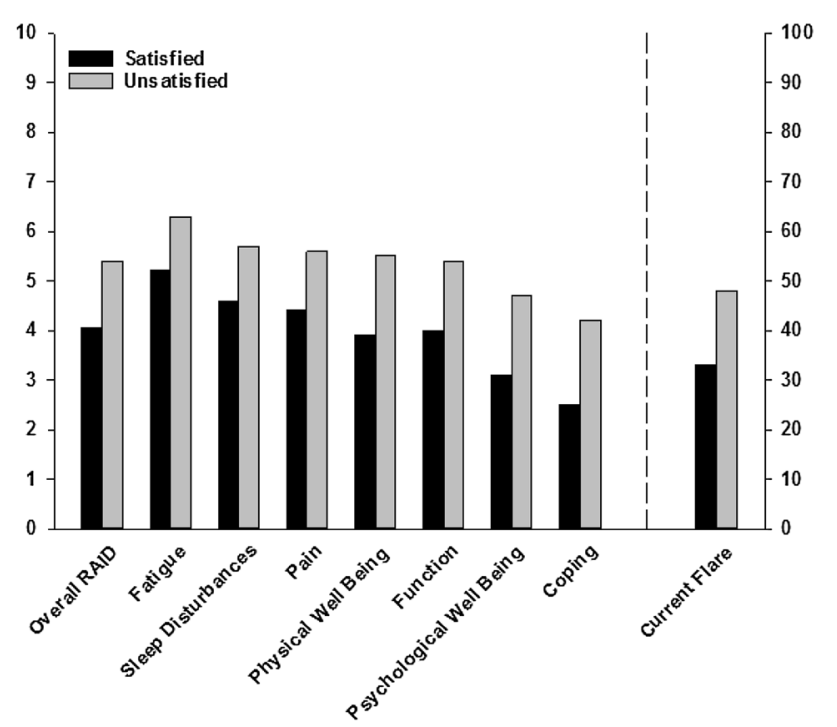

Fig. 2 Mean Rheumatoid Arthritis Impact Of Disease (RAID) score overall and by domain and flare status, stratified by patient treatment satisfaction [defined as treatment satisfaction questionnaire for medication
(TSQM) global satisfaction score $\geq 80$ ]. Twenty-six percent $(n=67)$ were classified as being satisfied with treatment and $74 \%(n=188)$ as unsatisfied

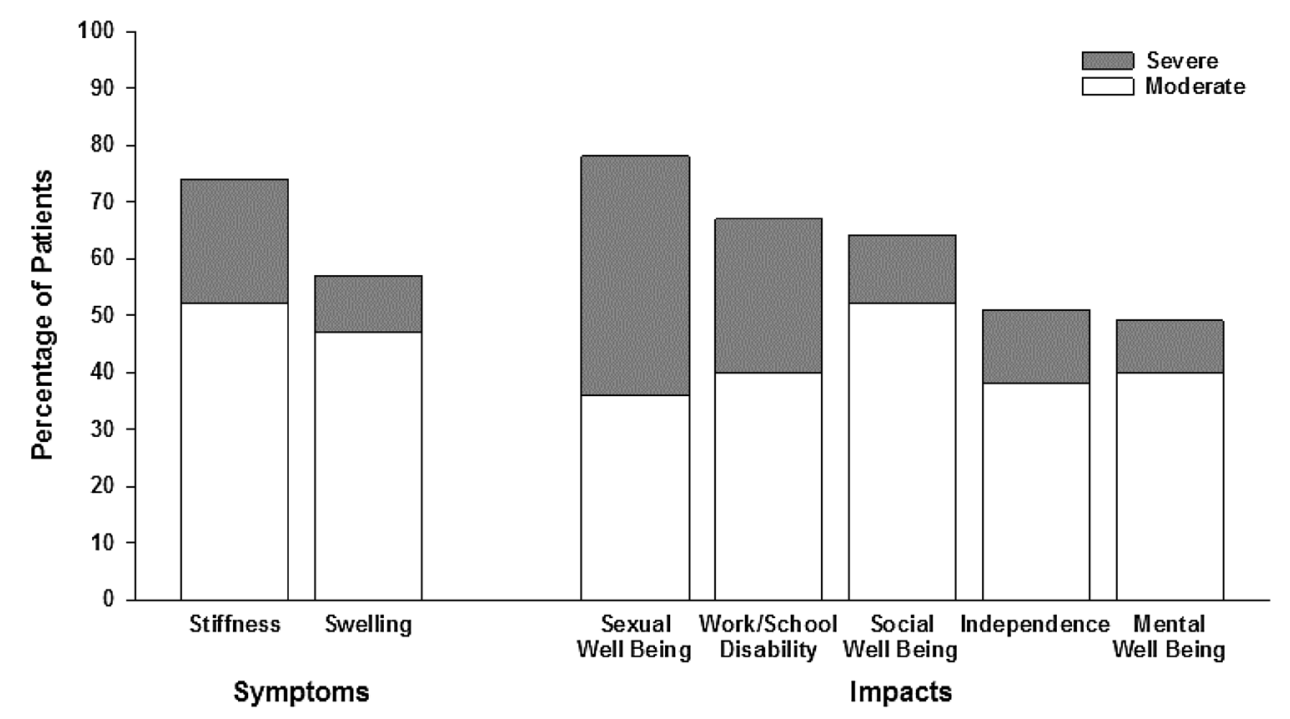

Fig. 3 Percentage of patients reporting moderate-to-severe symptoms and impacts

using a $0-10$ NRS (0 for no flare to 10 for the worst flare), the mean (SD) score was 5.8 (3.0).

\section{Subgroup Analyses of Treatment Satisfaction and RAID}

Current use of a bDMARD and prior use of another bDMARD were reported by 141 (55\%) patients. Compared with the overall sample, this subgroup of patients tended to report similar levels of treatment satisfaction (e.g., mean global satisfaction scores of 65 vs. 65 for the overall sample) and mean global and domain scores on the RAID as the overall patient population (Supplemental Table 3). 
Approximately half of the overall sample (53\%, $n=137)$ had been on their current RA medication for 1 to $<5$ years, and $29 \%(n=74)$ had been on medication for $\geq 5$ years. The latter subgroup of patients tended to report slightly higher scores on the RAID overall score and on individual domains compared with the overall sample (Supplemental Table 3).

Patients who were not satisfied with treatment, as defined by a TSQM global satisfaction score $<80$, reported higher scores on the RAID overall and across the seven domains than those who were satisfied (Fig. 2). Of note, patients who were satisfied with treatment tended to report moderate symptom severity in the overall and individual RAID domain scores. Additionally, among patients who were not satisfied with treatment, approximately half reported a current flare (Fig. 2).

\section{Choices}

When asked if there are enough medication choices available for themselves and their doctors to manage their RA, $36 \%(n=91)$ agreed or strongly agreed, $28 \%(n=71)$ were neutral, and $36 \%(n=90)$ disagreed or strongly disagreed.

\section{DISCUSSION}

In this real-world sample of patients with RA, the majority of patients had experience with a bDMARD, had been diagnosed with RA for over a year, and had been on their current RA treatment regimen for over a year, with $29 \%$ of patients having been on their treatment regimen for $\geq 5$ years. Despite this access and prolonged use of bDMARDs, many continued to experience bothersome symptoms and health impact (e.g., pain and impaired physical function), and almost half of the patients reported a flare. Using the TSQM global satisfaction score to assess treatment satisfaction, only a quarter of the patients in the overall sample were satisfied with treatment. Additionally, high proportions of patients indicated that they had not achieved remission or low disease activity with their current treatment, as assessed by RAID. The totality of evidence suggests unmet needs for the treatment of RA, including for currently bDMARD-treated patients.

Results from this study raise the question of why patients do not change their RA medications despite impaired function and disease activity, particularly in light of the treat-to-target strategy in which changing therapy is recommended if the designated target is not achieved [24]. The clinical inertia may derive from the differences between the physician's perception of disease activity and that of the patient [5]. As described above, the patient's pain and disability may derive from past joint structural damage and not be related to actual improper control of the disease. This survey, however, was not able to discriminate between pain from damage and pain from active disease. Prior studies have also hypothesized that the fear of medication side effects and a loss of control may be factors in the unwillingness to change [7, 8, 11, 25-27]. Furthermore, many patients do not want to change their therapy as long as their condition remains stable and does not worsen, even if a treatment change might result in them having improved QoL $[10,11]$. The current study also indicates that patients who had previous or current bDMARD experience reported similar treatment satisfaction compared with those who had not. One hypothesis for this is that patients with more extensive experience with treatment may become more accustomed to the positive effects of biologics over time; on the other hand, these results could indicate that patients with this experience and/or physicians have accepted some degree of symptoms and impairment [28].

The demographics of the study sample were representative of the population of patients with established RA in the United States. Notably, the majority of patients were female and white, with a mean age of 54.5 years $[11,29,30]$.

This study advances the current understanding of patient needs for their RA treatment. In particular, this study was conducted in a real-world setting, and patients directly reported about their RA treatment, care, symptoms, and impacts via a web-based survey. The simple, validated instruments, TSQM and RAID, were used to provide complimentary information on the patient experience. The TSQM 
provides insights into the patient experience with medication with respect to effectiveness, side effects, convenience, and global satisfaction, and the instrument may be used across a variety of medications and illnesses $[12,13]$. In contrast, the RAID was derived from a task force sponsored through the European League Against Rheumatism (EULAR), to measure the impact of RA on patient health and well-being [16]. The simplicity of both instruments ensured the feasibility of their administration over a web-based platform.

The prevalence of burdensome symptoms and adverse impacts on health-related QoL observed in this study suggests that there is unmet need for many patients despite the currently available treatment options, even among those patients who are satisfied with their RA treatment. Patient insights, such as those reported in the current study, can help physicians and patients discuss and decide upon a course of treatment; this shared decision-making is encouraged for the management of RA $[3,31]$. Furthermore, the patient perspective is important for drug development and study design, by identifying the symptoms and impacts of RA and treatment that are most important to patients but remain poorly addressed by currently available DMARDs. These include residual pain, fatigue, joint stiffness, sleep disturbances, sexual well-being, and other patient-reported outcomes; patient-centric outcome measures have only recently assumed greater prominence in the design of contemporary RA trials. Additionally, unmet needs are key elements of the US Food and Drug Administration's risk-benefit assessment framework [32].

Some findings from this study should be interpreted with caution. The generalizability of these results must be considered, given that this study enrolled a convenience sample of patients who were members of an online patient community group (CreakyJoints) or digital research registry (ArthritisPower ${ }^{\circledR}$ ). Because of this type of engagement, these patients may be not be representative of the broader group of RA patients, and RA and its variable manifestations may have a different impact on their overall health. Given that these patients are more likely to be healthseeking by virtue of their engagement with other patients and research participants, they may have greater interest in and access to newly available therapies. Despite this consideration, treatment changes were relatively infrequent. Likewise, as with most survey research, this study is subject to selection bias. Patients who participated in this survey may be different from those who did not participate. Additionally, patients self-reported their RA diagnosis and treatments, and there was no opportunity to confirm their responses (e.g., with medical record review); however, almost all (98\%) reported being under the care of a rheumatologist, and described current and/or past use of a bDMARD or csDMARD. The survey also may not have captured other factors, such as patient coping behavior or perceptions of illness, that have been shown to affect patient psychosocial outcomes [33]. Lastly, the survey also contained several questions that were not from validated instruments, which may affect the reliability and validity of responses.

\section{CONCLUSIONS}

This study demonstrates the significant unmet needs among patients with RA, despite the availability of current RA treatments and a high prevalence of past or current bDMARD use. Although many RA guidelines strongly encourage striving for remission, these findings suggest that many patients report much worse health states. Given that the perceived health state for patients who were satisfied with their RA treatments was relatively poor, acceptance of suboptimal disease control may be an impediment to appropriate RA treatment optimization; this has been suggested in recent publications in which patients have frequently refused to accelerate RA care despite active disease [26]. The patient perspective will be critical to make continual improvements in the treatment of RA and to encourage appropriate treatment switching and escalation.

\section{ACKNOWLEDGEMENTS}

The authors would like to thank the participants in the survey for their time and insights. 
All statements in this manuscript, including its findings and conclusions, are solely those of the authors and do not necessarily represent the views of PCORI, its Board of Governors, or its Methodology Committee.

Funding. Sponsorship for this study and the Rapid Service Fee were funded by Eli Lilly and Company (Indianapolis, IN, USA). This work was also partially supported through a PatientCentered Outcomes Research Institute (PCORI) award (PPRN-1306-04811). All authors had full access to all of the data in this study and take complete responsibility for the integrity of the data and accuracy of the data analysis. The sponsor only had access to summary tables.

Editorial Assistance. Editorial assistance in the preparation of this article was provided by Molly E. Tomlin of Eli Lilly and Company. This assistance was funded by Eli Lilly and Company.

Authorship. All named authors meet the International Committee of Medical Journal Editors (ICMJE) criteria for authorship for this article, take responsibility for the integrity of the work as a whole, and have given their approval for this version to be published.

Disclosures. Christine Radawski is an employee of Eli Lilly and Company. Carol L. Gaich is an employee of Eli Lilly and Company. Amy M. DeLozier is an employee of Eli Lilly and Company. Anabela Cardoso is an employee of Eli Lilly and Company. Mark C. Genovese has received grant/research support and has provided expert advice to Eli Lilly and Company and Novartis. Brett Haube has received grant/ research support from Eli Lilly and Company. W. Benjamin Nowell has received grant/research support from Eli Lilly and Company. Kelly Hollis has received grant/research support from Eli Lilly and Company. Kelly Gavigan has received grant/research support from Eli Lilly and Company. Maria Reynolds has received grant/research support from Eli Lilly and Company. Jeffrey R. Curtis has received grant/research support and provided expert advice to AbbVie, Amgen, BMS, Corrona, Eli Lilly and
Company, Janssen, Myriad, were consistent with the patient populations that appear in the literature Pfizer, Regeneron, Roche and UCB.

Compliance with Ethics Guidelines. The study was reviewed by the Schulman Institutional Review Board (IRB) and was determined to be exempt. The study was conducted in accordance with the ethical principles that have their origin in the Declaration of Helsinki and that are consistent with Good Pharmacoepidemiology Practices and applicable laws and regulations. Informed consent was obtained from all individual participants included in the study.

Data Availability. Lilly provides access to relevant anonymized patient-level data from studies on approved medicines and indications as defined by the sponsor-specific information on clinicalstudydatarequest.com. For details on submitting a request, see the instructions provided at clinicalstudydatarequest.com.

Open Access. This article is distributed under the terms of the Creative Commons Attribution-NonCommercial 4.0 International License (http://creativecommons.org/licenses/ by-nc/4.0/), which permits any noncommercial use, distribution, and reproduction in any medium, provided you give appropriate credit to the original author(s) and the source, provide a link to the Creative Commons license, and indicate if changes were made.

\section{REFERENCES}

1. Singh JA, Saag KG, Bridges SL Jr, et al. 2015 American College of Rheumatology guideline for the treatment of rheumatoid arthritis. Arthritis Rheumatol. 2016;68:1-26.

2. Smolen JS, Aletaha D. Rheumatoid arthritis therapy reappraisal: strategies, opportunities and challenges. Nat Rev Rheumatol. 2015;11:276-89.

3. Smolen JS, Landewe R, Breedveld FC, et al. EULAR recommendations for the management of rheumatoid arthritis with synthetic and biological disease-modifying antirheumatic drugs: 2013 update. Ann Rheum Dis. 2014;73:492-509. 
4. Taylor PC, Moore A, Vasilescu R, Alvir J, Tarallo M. A structured literature review of the burden of illness and unmet needs in patients with rheumatoid arthritis: a current perspective. Rheumatol Int. 2016;36:685-95.

5. Strand V, Wright GC, Bergman MJ, Tambiah J, Taylor PC. Patient expectations and perceptions of goal-setting strategies for disease management in rheumatoid arthritis. J Rheumatol. 2015;42:2046-54.

6. Fraenkel L, Nowell WB, Michel G, Wiedmeyer C. Preference phenotypes to facilitate shared decisionmaking in rheumatoid arthritis. Ann Rheum Dis. 2018;77:678-83.

7. Funahashi K, Matsubara T. What RA patients expect of their treatment-discussion over the result of our survey. Clin Rheumatol. 2012;31:1559-66.

8. Gonzalez CM, Carmona L, de Toro J, et al. Perceptions of patients with rheumatic diseases on the impact on daily life and satisfaction with their medications: RHEU-LIFE, a survey to patients treated with subcutaneous biological products. Patient Prefer Adherence. 2017;11:1243-52.

9. Poulos C, Hauber AB, Gonzalez JM, Turpcu A. Patients' willingness to trade off between the duration and frequency of rheumatoid arthritis treatments. Arthritis Care Res. 2014;66:1008-15.

10. Takahashi N, Sasaki K, Nishiyama T, Naniwa $T$. Satisfaction and attitudes toward therapy in patients with rheumatoid arthritis. Mod Rheumatol. $2012 ; 22: 376-81$.

11. Wolfe F, Michaud K. Assessment of pain in rheumatoid arthritis: minimal clinically significant difference, predictors, and the effect of anti-tumor necrosis factor therapy. J Rheumatol. 2007;34:1674-83.

12. Atkinson MJ, Kumar R, Cappelleri JC, Hass SL. Hierarchical construct validity of the treatment satisfaction questionnaire for medication (TSQM version II) among outpatient pharmacy consumers. Value Health. 2005;8(Suppl 1):S9-24.

13. Atkinson MJ, Sinha A, Hass SL, et al. Validation of a general measure of treatment satisfaction, the Treatment Satisfaction Questionnaire for Medication (TSQM), using a national panel study of chronic disease. Health Qual Life Outcomes. 2004;2:12.

14. Bharmal M, Payne K, Atkinson MJ, Desrosiers MP, Morisky DE, Gemmen E. Validation of an abbreviated Treatment Satisfaction Questionnaire for Medication (TSQM-9) among patients on antihypertensive medications. Health Qual Life Outcomes. 2009;7:36.
15. Reynolds K, Viswanathan HN, O'Malley CD, et al. Psychometric properties of the Osteoporosis-specific Morisky Medication Adherence Scale in postmenopausal women with osteoporosis newly treated with bisphosphonates. Ann Pharmacother. 2012;46:659-70.

16. Gossec L, Dougados M, Rincheval N, et al. Elaboration of the preliminary Rheumatoid Arthritis Impact of Disease (RAID) score: a EULAR initiative. Ann Rheum Dis. 2009;68:1680-5.

17. Dougados M, Brault $Y$, Logeart I, van der Heijde D, Gossec L, Kvien T. Defining cut-off values for disease activity states and improvement scores for patient-reported outcomes: the example of the Rheumatoid Arthritis Impact of Disease (RAID). Arthritis Res Ther. 2012;14:R129.

18. Salaffi F, Di Carlo M, Vojinovic J, et al. Validity of the rheumatoid arthritis impact of disease (RAID) score and definition of cut-off points for disease activity states in a population-based European cohort of patients with rheumatoid arthritis. Jt Bone Spine. 2018;85:317-22.

19. Jenkins HH, Spencer ED, Weissgerber AJ, Osborne LA, Pellegrini JE. Correlating an 11-point verbal numeric rating scale to a 4-point verbal rating scale in the measurement of pruritus. J Perianesthesia Nurs. 2009;24:152-5.

20. Kuettel D, Primdahl J, Christensen R, Ornbjerg LM, Horslev-Petersen K. Impact of patient-reported flares on radiographic progression and functional impairment in patients with rheumatoid arthritis: a cohort study based on the AMBRA trial. Scand J Rheumatol. 2018;47:87-94.

21. Ometto F, Raffeiner B, Bernardi L, et al. Self-reported flares are predictors of radiographic progression in rheumatoid arthritis patients in 28-joint disease activity score remission: a 24-month observational study. Arthritis Res Ther. 2016;18:89.

22. Gossec L, Paternotte S, Aanerud GJ, et al. Finalisation and validation of the rheumatoid arthritis impact of disease score, a patient-derived composite measure of impact of rheumatoid arthritis: a EULAR initiative. Ann Rheum Dis. 2011;70:935-42.

23. Hunter TM, Boytsov NN, Zhang X, Schroeder K, Michaud K, Araujo AB. Prevalence of rheumatoid arthritis in the United States adult population in healthcare claims databases, 2004-2014. Rheumatol Int. 2017;37:1551-7.

24. Smolen JS, Breedveld FC, Burmester GR, et al. Treating rheumatoid arthritis to target: 2014 update of the recommendations of an international task force. Ann Rheum Dis. 2016;75:3-15. 
25. Barton JL. Patient preferences and satisfaction in the treatment of rheumatoid arthritis with biologic therapy. Patient Prefer Adherence. 2009;3: 335-44.

26. Harrold LR, Reed GW, John A, et al. Cluster-randomized trial of a behavioral intervention to incorporate a treat-to-target approach to care of US patients with rheumatoid arthritis. Arthritis Care Res. 2018;70:379-87.

27. Kaufmann J, Feist E, Roske AE, Schmidt WA. Monotherapy with tocilizumab or TNF-alpha inhibitors in patients with rheumatoid arthritis: efficacy, treatment satisfaction, and persistence in routine clinical practice. Clin Rheumatol. 2013;32:1347-55.

28. van den Reek JM, van Luumig PP, Otero ME, et al. Satisfaction of treatment with biologics is high in psoriasis: results from the Bio-CAPTURE network. Br J Dermatol. 2014;170:1158-65.
29. Goel N, Cummins G, Downing J, van Tuyl L. FRI0362 rapid acquisition of data on the patient perspective in rheumatoid arthritis through a digital portal. Ann Rheum Dis. 2015;74:557.

30. $\mathrm{Xu} \mathrm{B}$, Lin J. Characteristics and risk factors of rheumatoid arthritis in the United States: an NHANES analysis. Peer J. 2017;5:e4035.

31. Fries JF, Spitz P, Kraines RG, Holman HR. Measurement of patient outcome in arthritis. Arthritis Rheum. 1980;23:13-45.

32. Califf RM. Benefit-risk assessments at the US food and drug administration: finding the balance. JAMA. 2017;317:693-4.

33. Van der Elst K, Verschueren P, Stouten V, et al. Patient reported outcome data from the Care in early rheumatoid arthritis trial: opportunities for broadening the scope of treating to target. Arthritis Care Res. 2019;5:5. https://doi.org/10.1002/acr.23900. 